\title{
High Serum IgE Concentration in Patients with Psoriasis
}

\author{
Caifeng Chen, Xuerui Zheng, Qiqi Duan, Peiwen Yang and Yan Zheng* \\ Department of Dermatology, the Second Affiliated Hospital, School of Medicine, Xi'an Jiaotong University, China
}

Received: July 05, 2017; Accepted: August 16, 2017; Published: August 22, 2017

"Corresponding author: Yan Zheng, Department of Dermatology, the Second Affiliated Hospital, School of Medicine, Xi'an Jiaotong University, 157 Xiwu Road, Xi'an 710004, China, Tel: 15319931238, E-mail: zenyan66@126.com

\begin{abstract}
Objectives: To compare the expression levels of serum IgE between patients with psoriasis and normal controls and to analyze its association with gender, age, disease duration, types of psoriasis and some circulating cytokines in psoriasis.
\end{abstract}

Methods: Serum levels of IgE, IL-6, IL-8 and TNF- $\alpha$ were measured by enzyme-linked immunosorbent assay.

Results: The serum IgE levels in patients with psoriasis were significantly higher than those in the control group $(\mathrm{P}<0.01)$. Furthermore, mean serum IgE concentration in psoriatic erythroderma was significantly higher than that in psoriasis vulgaris. No correlation between age, gender or disease duration and serum IgE level was observed. Correlation analysis showed that the serum IgE levels were positively associated with the levels of serum TNF- $\alpha(\mathrm{r}=$ $0.4428, \mathrm{P}<0.001$ ) rather than IL-6 or IL-8.

Conclusions: The increased expression of IgE maybe involved in the pathogenesis of psoriasis and it could be a promising target for psoriasis treatment.

Keywords: Psoriasis; IgE; TNF- $\alpha$; Case-Control study;

\section{Introduction}

Psoriasis is a common chronic skin disorder characterized by abnormal epidermal proliferation and infiltration of inflammatory cells which affects approximately 2-4 \% of the general population [1-3] Psoriasis Vulgaris (PV), the most common clinical variant, comprises 85 to $90 \%$ of all patients with psoriasis. This disease is usually manifested as well-demarcated erythematous oval plaques with silvery scale on extensor surfaces. Pustular psoriasis (PP) is an uncommon but potentially lifethreatened clinical variant that presents with multiple sterile pustules on erythematous background. Erythrodermic Psoriasis (PE) is a variant that may affect all body sites and involves less than $2 \%$ of all psoriatic patients. Psoriatic Arthritis (PsA) is characterized by pain, swelling and tenderness of the involved joints, which may lead to joint damage and disability in the end. Several studies have validated that psoriasis is associated with multiple comorbidities and greatly reduces the quality of patients' life by impacting patients psychologically and physically $[4,5]$. However, the pathogenesis of psoriasis is complex and remains poorly understood [6].
Psoriasis is generally thought to be an immune-mediated inflammatory skin disease and IgE plays an important role in innate and acquired immunity. There is some evidence that IgE may take a part in the pathogenesis of psoriasis. Some studies found increased levels of serum IgE in psoriasis patients while other studies showed that the serum IgE levels in patients with psoriasis are no different to that of the normal controls [7-13]. The purpose of our study was to measure the expression of serum IgE in patients with psoriasis and normal subjects, and to analyze its association with sex, age, duration, types of psoriasis and some circulating cytokines.

\section{Methods and Materials}

\section{Patients and controls}

A case-control study was performed and all patients were from Department of Dermatology of the Second Affiliated Hospital of Xi'an Jiaotong University. Totally 98 patients with psoriasis, including 60 cases of PV, 15 cases of PE, 19 PP, 4 cases of PsA and 25 healthy controls with matched age and sex were enrolled in this study. Patients with history of atopic dermatitis, allergic diseases, parasitic diseases or systemic disease were excluded. Patients with administration of corticosteroids or immunemodulators were also excluded. The informed consent was obtained from all participants and the study was approved by the ethical committee of the Second Affiliated Hospital of Xi'an Jiaotong University.

\section{Detection of serum IgE and cytokines}

Serum levels of IgE, IL-6, IL-8 and TNF- $\alpha$ were measured by ELISA using commercially available kits (BD Biosciences, USA), according to the manufacturers' instructions. The normal range for serum IgE was 0-100 $\mathrm{IU} / \mathrm{ml}$ with this assay.

\section{Statistical analysis}

Statistical analysis was performed using GraphPad Prism 5.0 (La Jolla, CA, USA) and the SPSS 21.0 software (SPSS Inc, Chicago, IL). Data were expressed as mean $\pm \mathrm{SD}$. Student's t-test or $\mathrm{X}^{2}$-test was used for Statistical comparisons. The Spearman's rank test was used in correlation analyses. $\mathrm{p}<0.05$ was considered statistically significant. 


\section{Results}

\section{Serum IgE levels in psoriasis patients}

The serum IgE level was elevated in $53.06 \%$ of total patients with psoriasis compared to $12 \%$ of the control group (Figure 1A). Serum IgE levels were significantly higher in psoriatic patients than those in controls $\left(X^{2}=13.585, P<0.001\right)$. Mean serum IgE concentrations was $237.01 \pm$
$335.45 \mathrm{IU} / \mathrm{ml}$ (from 4.74 to $1490 \mathrm{IU} / \mathrm{ml}$ ) in the patient group versus 46.59 $\pm 37.23 \mathrm{IU} / \mathrm{ml}$ (from 4.74 to $129 \mathrm{IU} / \mathrm{ml}$ ) in the healthy control group. The difference was statistically significant $(\mathrm{P}<0.01$, Figure $1 \mathrm{~B})$. The Mean serum IgE concentration was $247.97 \pm 328.93 \mathrm{IU} / \mathrm{ml}$ (from 6.24 to 1480 $\mathrm{IU} / \mathrm{ml}$ ) in male patients and $214.42 \pm 352.78 \mathrm{IU} / \mathrm{ml}$ (from 4.74 to 1490 $\mathrm{IU} / \mathrm{ml}$ ) in female patients respectively. However, no statistical difference was found between the two groups (Figure 1C).

A
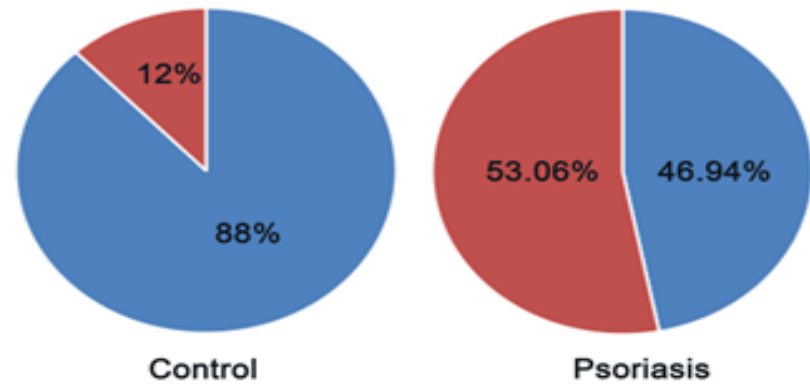

B

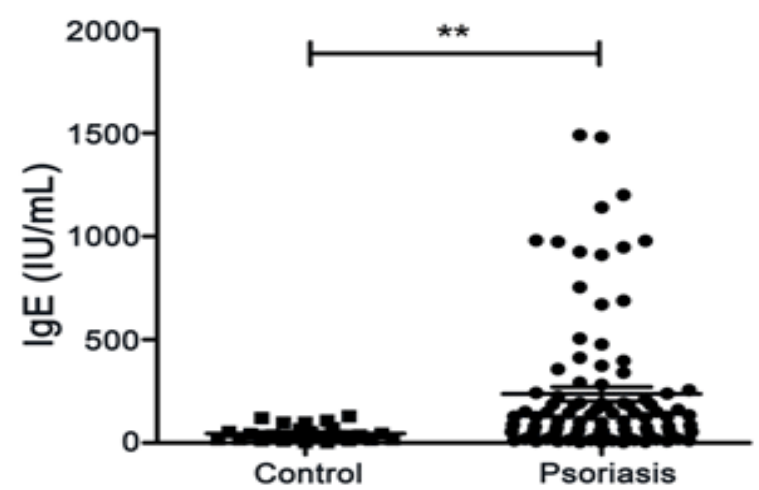

C

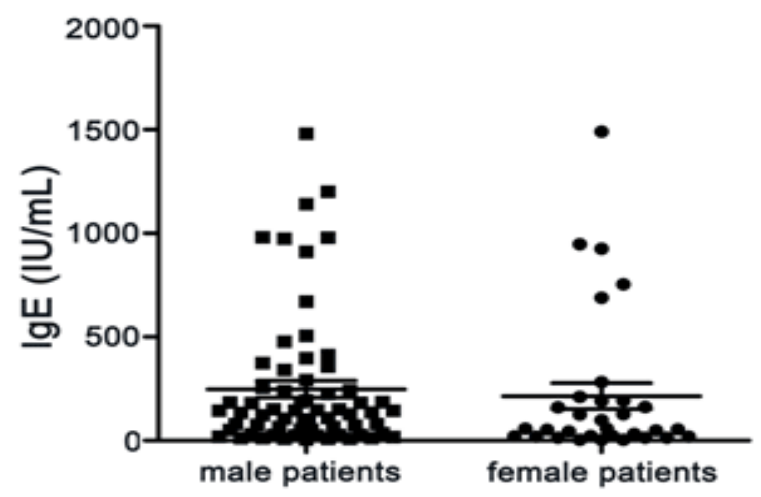

Figure 1: Serum IgE levels in psoriasis. (A) Serum IgE concentrations was increased in $53.06 \%$ of patients with psoriasis compared to $12 \%$ of healthy controls. (B) Mean serum IgE levels in psoriasis were significantly higher than those in controls. (C) No statistical difference was observed on mean serum IgE levels from male patients compared with female patients. ${ }^{*} \mathrm{P}<0.05,{ }^{* *} \mathrm{P}<0.01,{ }^{* * *} \mathrm{P}<0.001$.

\section{Correlation of IgE concentration with age, duration and types of Correlation of IgE level with circulating cytokines} psoriasis

The disease duration of psoriatic patients in this study varied from 1 to 720 months. Patients' mean age was $40.06 \pm 17.34$ years. There was no correlation between age $(\mathrm{p}>0.05)$ or disease duration $(\mathrm{P}>0.05)$ and serum IgE level. All types of psoriasis had statistically significant elevation in the serum IgE levels compared to the controls with PE showing the highest elevation. Statistical difference of IgE concentration was also observed between PE and PV (Figure 2).

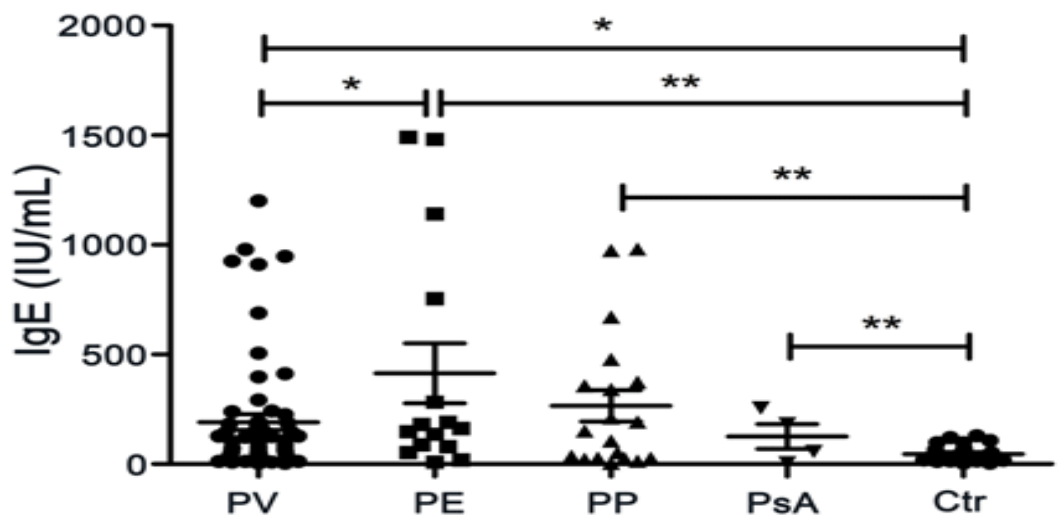

Figure 2: Serum IgE levels in types of psoriasis. ${ }^{*} \mathrm{P}<0.05$, ${ }^{* *} \mathrm{P}<0.01,{ }^{* * *} \mathrm{P}<$ 0.001 .
We observed that the serum IgE levels were positively correlated with the serum TNF- $\alpha$ levels in psoriasis patients (Figure 3A). However, no correlation was found between the levels of serum IgE and IL-6 (Figure 3B) or IL-8 levels (Figure 3C).

\section{Discussion}

Humoral changes that elevated serum IgA, IgG and antinuclear antibodies in psoriasis have been demonstrated within the past years $[14,15]$. However, studies on serum IgE's expression in psoriasis are limited and results are paradoxical. IgE is produced by B lymphocytes and the production is regulated by $\mathrm{T}$ lymphocytes. IgE is generally acknowledged as a typical mediator of allergic response, which is low in healthy subjects and elevated in atopic conditions, such as eczema, rhinitis and asthma [16,17]. IgE recognizes exogenous antigens and signals through Fce receptors (FceRs), including FceR I and FceR II, triggering an immunologic response. Moreover, it has been found that the levels of serum IgE significantly increase in patients with systemic lupus erythematosus, alopecia areata and other autoimmune disease $[18,19]$.

Our data clearly demonstrated that the elevation in the serum IgE level was more frequently found in patients with psoriasis (53.06\%) than in healthy controls (12\%). The result is consistent with some studies 
A

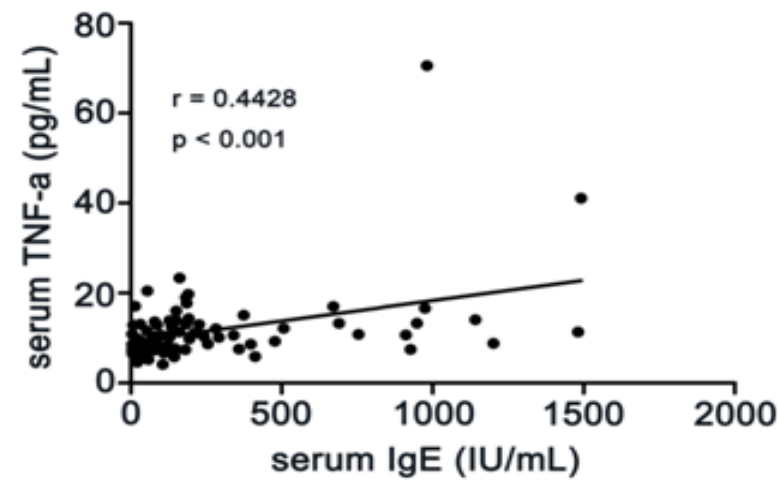

B

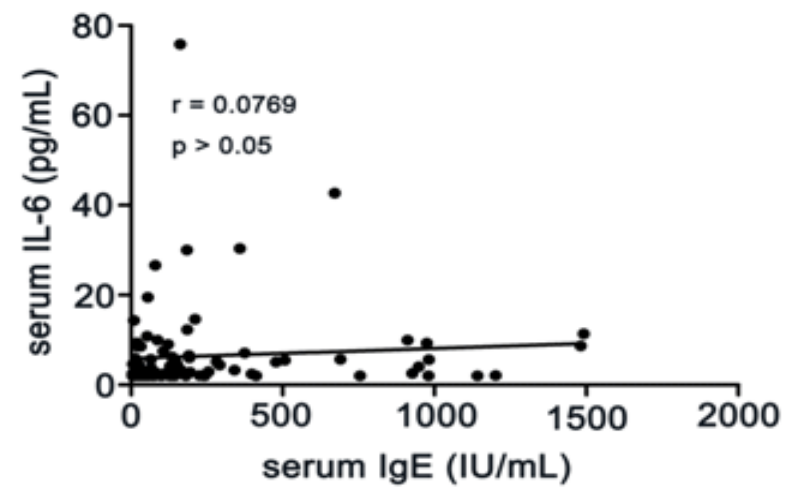

C

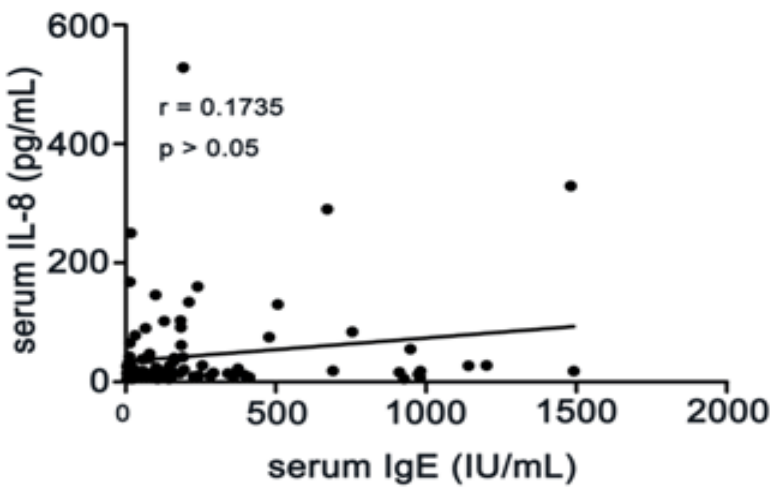

Figure 3: Correlation between serum IgE levels and circulating cytokines in psoriatic patients. (A) Correlation of IgE with TNF- $\alpha$ serum levels in psoriasis. (B) Correlation of IgE with IL-6 serum levels in psoriasis. (C) Correlation of IgE with IL-8 serum levels in psoriasis.

[7-11]. Yan et al demonstrated that 39\% of patients with psoriasis had elevated serum IgE concentrations and they observed that lesional skin of patients with psoriasis contains more IgE+ and FceRI+ cells [20]. Chen et al reported that the serum IgE level was elevated in $46 \%$ psoriatic patients [11]. In addition, our findings were similar to a clinical study, which showed that the serum IgE concentration was increased in $42 \%$ psoriatic patients and observed that there existed no correlation between age, gender or disease duration and total serum IgE concentration [8]. Moreover, we demonstrated that all types of psoriasis had significantly increased serum IgE levels compared to the controls. In agreement with our data, a case-control study showed that the mean level of serum IgE in PE patients was much higher than that in PV patients and they suggested that a shift from Th1 to Th2 occurred in PE [10]. The IgE levels were also higher in PsA patients in our study, but the difference between PsA and PV was not statistically significant. Nevertheless, a retrospective study observed that psoriatic patients with joint symptoms had higher IgE levels and another study found completely opposite result [21,22]. Our data were also in contrast to other researches showing no increase of IgE levels in psoriasis $[12,13]$.

Nevertheless, the mechanisms of IgE upregulation in psoriasis are still poorly understood. IgE is usually dominated by Th2 cytokines and Th2 cytokines IL-13 and IL-4 mediate class-switching towards IgE in atopic diseases [23,24]. However, IL-13 and IL-4 cannot be produced by keratinocytes and IL-13 and IL-4 were downregulated in psoriasis. There may be other mechanisms promoting the over-production of IgE in psoriasis. Psoriasis is an immune-mediated inflammatory skin disorder and there is mounting evidence that levels of TNF- $\alpha$, IL-6, IL-8 and IL-17, as crucial cytokines involved in psoriais's pathogenesis, are elevated in psoriasis [3]. Previous studies showed that IL-8 inhibited IL-4-induced IgE production and endogenous TNF- $\alpha$ and IL- 6 are essential for IgE production in atopic patients [25,26]. In addition, IgE promotes the autocrine production of IL-6 in human lung mast cells [27]. Recently, a study showed that IgE-secreting cells differentiation and IgE production could be directly promoted by IL-17 [28]. We wondered that whether TNF- $\alpha$, IL- 6 or IL- 8 could be, just like IL-17, involved in the regulation of IgE in psoriasis, so we measured the serum levels of TNF- $\alpha$, IL- 6 and IL-8 in patients with psoriasis and performed correlation analysis. Although no significant correlation was found between IL-6 or IL-8 and IgE serum levels, our study detected positive correlation between circulating TNF- $\alpha$ levels and IgE serum levels in psoriasis.

There exist some limitations in this study. The current study is a single center study and the sample size is relatively small, particular the sample size of PsA. We only detected the total IgE levels in patients with psoriasis, but did not measure the levels of the specific IgE levels to common allergens.

In conclusion, our data showed that serum IgE level was highly expressed in psoriasis, particularly in psoriatic erythroderma, indicating that the high serum IgE concentration may be a common feature in patients with psoriasis. The exact role that IgE plays in the pathogenesis of psoriasis should be further studied. Correlation analysis showed that serum IgE levels was positively associated with serum TNF- $\alpha$. However, whether there is a causal link between TNF- $\alpha$ and IgE and if so, their relative roles in the causal relationship remain to be clarified.

\section{Acknowledgments}

This work was supported by grants from the National Natural Science Foundation of China $(81573055,81371732)$ and partially supported by Funds of Shaanxi Province (2015KTCL03-10) and 2nd Hospital of Xi'an Jiaotong University. 


\section{References}

1. Boehncke WH, Schon MP. Psoriasis. The Lancet. 2015;386(9997):983-994. DOI: ttp:// dx.doi.org/10.1016/S0140-6736(14)61909-7

2. Nestle FO, Kaplan DH, Barker J. Psoriasis. N Engl J Med. 2009;361(5):496-509. doi: 10.1056/NEJMra0804595

3. Mahil SK, Capon F, Barker JN. Update on psoriasis immunopathogenesis and targeted immunotherapy. Semin Immunopathol. 2016;38(1):11-27. doi: 10.1007/s00281-0150539-8

4. Gelfand JM, Yeung H. Metabolic syndrome in patients with psoriatic disease. J Rheumatol Suppl. 2012;89:24-28. doi: 10.3899/jrheum.120237

5. Ryan C, Kirby B. Psoriasis is a systemic disease with multiple cardiovascular and metabolic comorbidities. Dermatol Clin. 2015;33(1):41-55. doi: 10.1016/j. det.2014.09.004

6. Harden JL, Krueger JG, Bowcock AM. The immunogenetics of Psoriasis: A comprehensive review. J Autoimmun. 2015;64: 66-73. doi: 10.1016/j.jaut.2015.07.008

7. Ding Y, Yi X, Yu N. Serum IgE levels are increased in patients with generalized pustular psoriasis. Clin Exp Dermatol. 2013;38(5):549-552. doi: 10.1111/ced.12086

8. Ovcina-Kurtovic N, Kasumagic-Halilovic E. Serum levels of total immunoglobulin E in patients with psoriasis: relationship with clinical type of disease. Med Arh. 2010;64(1):28-29.

9. Paparo SB, Guaragna MA, Albanesi M. High IgE levels in patients affected by psoriasis: review of the literature and personal observations. Clin Ter. 2014;165(2):91-93. doi: 10.7471/CT.2014.1682

10.Li LF, Sujan SA, Yang H, Wang WH. Serum immunoglobulins in psoriatic erythroderma Clin Exp Dermatol. 2005;30(2):125-127.

11.Chen ZY, Ainsworth SK, Khan T, Pilia PA, Dobson RL. Immunoglobulin E in psoriasis evaluated by paper radioimmunosorbent and paper enzymeimmunosorbent tests. Acta Derm Venereol. 1985;65(1):14-18.

12.Lajevardi V, Ghiasi M, Goodarzi A, Mohtasham S, Ansari M, Hedayat K, et al. Total serum IgE concentration in patients with psoriasis: a case-control study. Acta Med Iran. 2014;52(7):515-518.

13.Guilhou JJ, Clot J, Meynadier J, Lapinskin H. Immunological aspects of psoriasis. Br Dermatol. 1976;94(5):501-507.

14.Kulick KB, Mogavero H Jr, Provost TT, Reichlin M. Serologic studies in patients with lupus erythematosus and psoriasis. J Am Acad Dermatol. 1983;8(5):631-634.
15.Singh S, Singh U, Singh S. Prevalence of autoantibodies in patients of psoriasis. J Clin Lab Anal. 2010;24(1):44-48. doi: 10.1002/jcla.20365

16.Sutton BJ, Gould HJ. The human IgE network. Nature. 1993;366(6454):421-428.

17.Gurevitch AW, Heiner DC, Reisner RM. IgE in atopic dermatitis and other common dermatoses. Arch Dermatol. 1973;107(5):712-715. doi:10.1001/ archderm.1973.01620200030006

18.Mikecz K, Sonkoly I, Meszaros C, Szegedi G. Serum IgE level in systemic lupus erythematosus. Acta Med Hung. 1985;42(1-2):59-65.

19.Kasumagic-Halilovic E. Serum levels of total IgE in patients with alopecia areata: Relationship with clinical type of the disease. Acta Dermatovenerol Croat. 2006;14(3):149-152.

20.Yan KX, Huang Q, Fang X, Zhang ZH, Han L, Gadaldi K, et al. IgE and FceRI are highly expressed on innate cells in psoriasis. Br J Dermatol. 2016;175(1):122-133. doi: 10.1111/bjd.14459

21.Voulgari PV, Gaitanis G, Fidhi L, Migkos MP, Bassukas ID. Joint complaints correlate with increased serum IgE levels in patients hospitalized for moderate-to-severe psoriasis: A single center retrospective study. J Am Acad Dermatol. 2016;74(5):1014-1015.e4. doi: 10.1016/j.jaad.2015.11.026

22.Hajdarbegovic E, Nijsten T, Westgeest A, Habraken F, Hollestein L, Thio B. Decreased prevalence of atopic features in patients with psoriatic arthritis, but not in psoriasis vulgaris. J Am Acad Dermatol. 2013;68(2):270-277. doi: 10.1016/j. jaad.2012.07.018

23.Worm M, Henz BM. Molecular regulation of human IgE synthesis. J Mol Med (Berl). 1997;75(6):440-447.

24.Geha RS, Jabara HH, Brodeur SR. The regulation of immunoglobulin E class-switch recombination. Nat Rev Immunol. 2003;3(9):721-732.

25.Kimata H, Lindley I, Furusho K. Selective inhibition of spontaneous IgE and IgG4 production by interleukin-8 in atopic patients. Blood. 1995;85(11):3191-3198.

26.Kimata H, Yoshida A, Ishioka C, Lindley I, Mikawa H. Interleukin 8 (IL-8) selectively inhibits immunoglobulin E production induced by IL-4 in human B cells. J Exp Med. 1992;176(4):1227-1231.

27.Cruse G, Cockerill S, Bradding P. IgE alone promotes human lung mast cell survival through the autocrine production of IL-6. BMC Immunol. 2008;9:2. doi: 10.1186/14712172-9-2

28.Milovanovic M, Drozdenko G, Weise C, Babina M, Worm M. Interleukin-17A promotes IgE production in human B cells. J Invest Dermatol. 2010;130(11):2621-2628. doi: 10.1038/jid.2010.175 